\title{
Chiral Silanes via Asymmetric Hydrosilylation with Catalytic CuH
}

Bruce H. Lipshutz, ${ }^{* a}$ Naoki Tanaka, ${ }^{a}$ Benjamin R. Taft, ${ }^{a}$ Ching-Tien Lee

${ }^{a}$ Department of Chemistry and Biochemistry

University of California

Santa Barbara, CA 93106 USA

Supporting Information 
General. Reactions were performed in oven-dried glassware under an argon atmosphere containing a Teflon coated stir bar and dry septum. THF and toluene were freshly distilled from $\mathrm{Na} / \mathrm{benzophenone}$ ketyl prior to use. All commercially available reagents were distilled either from $\mathrm{CaH}_{2}$ or molecular sieves under an inert atmosphere before use. $(R, S)$-PPF-P $(t-\mathrm{Bu})_{2}$ was generously supplied by Solvias. (R)-(-)-DTBM-SEGPHOS was generously provided by Takasago International Corp. GC analyses were carried out using an HP-5 capillary column $(0.25 \mu \times 30 \mathrm{~m}$; cross-linked 5\% PHME siloxane $)$ and a time program beginning with $5 \mathrm{~min}$ at $50{ }^{\circ} \mathrm{C}$ followed by $20{ }^{\circ} \mathrm{C} / \mathrm{min}$ ramp to $280{ }^{\circ} \mathrm{C}$, then $20 \mathrm{~min}$ at this temp. Column chromatography was performed using Davisil Grade 633 Type 60A silica gel. TLC analyses were performed on commercial Kieselgel 60 F254 silica gel plates. NMR spectra were obtained on Varian Inova systems using $\mathrm{CDCl}_{3}$ as solvent, with proton and carbon resonances at $400 \mathrm{MHz}$ and 100 $\mathrm{MHz}$, respectively. FTIR spectra were obtained on an ATI Mattson Infinity series spectrometer neat on $\mathrm{NaCl}$ plates or KBR pellets and are reported in $\mathrm{cm}^{-1}$. Mass spectral data were acquired on a VF Autospec or an analytical VG-70-250 HF instrument.

Representative Procedure for the catalytic asymmetric $\mathrm{CuH}$ hydrosilylation of $\boldsymbol{\beta}$-silyl enoates. (S)-Ethyl 3-(dimethylphenylsilyl)butanoate (Table 1, entry 1a); Method A. To a $50 \mathrm{~mL}$ round bottomed flask, flame dried and purged with argon, was added $\mathrm{CuCl}(1.5 \mathrm{mg}, 0.015 \mathrm{mmol}),(R, S)-\mathrm{PPF}-$ $\mathrm{P}(t-\mathrm{Bu})_{2}(2.7 \mathrm{mg}, 0.005 \mathrm{mmol})$, and $\mathrm{NaO}-t-\mathrm{Bu}(1.4 \mathrm{mg}, 0.015 \mathrm{mmol})$. Toluene $(1 \mathrm{~mL})$ was added and the solution was stirred at $0{ }^{\circ} \mathrm{C}$ for $30 \mathrm{~min}$, followed by addition of $t$ - BuOH $(52 \mu \mathrm{L}, 0.55 \mathrm{mmol})$. The solution was cooled to $-30{ }^{\circ} \mathrm{C}$ before addition of PMHS $(65 \mu \mathrm{L}, 1.0 \mathrm{mmol})$ and was further stirred at $-30{ }^{\circ} \mathrm{C}$ for $10 \mathrm{~min}$. Enoate $(124 \mathrm{mg}, 0.50 \mathrm{mmol})$ was added via syringe. The mixture was stirred at -30 ${ }^{\circ} \mathrm{C}$ until complete by TLC $(9 \mathrm{~h}$; ether/hexanes $=1 / 9)$. The reaction was quenched with sat. aq. $\mathrm{NaHCO}_{3}$ and diluted with ether. The aqueous layer was extracted with ether and the organic layer was washed with brine, dried over anhydrous $\mathrm{MgSO}_{4}$, filtered, and concentrated by rotary evaporation. The residue was purified by flash chromatography (ether/hexanes $=1 / 10)$ to afford the title product $(120 \mathrm{mg}, 96 \%)$ as a clear oil. The de was determined by ${ }^{1} \mathrm{H}$ NMR of the corresponding imide derivative to be $95 \%$. $\mathrm{R}_{\mathrm{f}}$ $=0.36$ (ether/hexanes = 1/9); IR (neat): 3070, 2956, 1734, 1428, 1368, $1208 \mathrm{~cm}^{-1} ;{ }^{1} \mathrm{H} \mathrm{NMR}(400 \mathrm{MHz}$, $\left.\mathrm{CDCl}_{3}\right) \delta 0.29(\mathrm{~s}, 6 \mathrm{H}), 0.98(\mathrm{~d}, J=7.4 \mathrm{~Hz}, 3 \mathrm{H}), 1.24(\mathrm{t}, J=7.1 \mathrm{~Hz}, 3 \mathrm{H}), 1.45(\mathrm{~m}, 1 \mathrm{H}), 2.05(\mathrm{dd}, J=$ 15.2, 11.4 Hz, 1H), 2.38 (dd, $J=15.2,4.0 \mathrm{~Hz}, 1 \mathrm{H}), 4.09$ (q, $J=7.1 \mathrm{~Hz}, 2 \mathrm{H}), 7.33-7.40$ (m, 3H), 7.47$7.54(\mathrm{~m}, 2 \mathrm{H}) ;{ }^{13} \mathrm{C}$ NMR $\left(100 \mathrm{MHz}, \mathrm{CDCl}_{3}\right) \quad \delta-5.14,-4.84,14.44,14.63,16.66,37.04,60.37,127.96$, 129.26, 134.11, 137.49, 174.14; EIMS m/z (\%): 250(5.4)[ $\left.\mathrm{M}^{+}\right], 235(23), 205(16), 145(27), 135(100)$; HREIMS calcd for $\mathrm{C}_{14} \mathrm{H}_{22} \mathrm{O}_{2} \mathrm{Si}$ : 250.1389; found $250.1390\left[\mathrm{M}^{+}\right]$.

(Table 1, entry 1b) Method B. To a $50 \mathrm{~mL}$ round bottomed flask, flame dried and purged with argon, was added $\left(\mathrm{Ph}_{3} \mathrm{P}\right) \mathrm{CuH}(4.9 \mathrm{mg}, 0.015 \mathrm{mmol})$ and $(R)$-DTBM-SEGPHOS $(5.9 \mathrm{mg}, 0.005 \mathrm{mmol})$. Toluene $(1 \mathrm{~mL})$ was added and the solution cooled to $-30{ }^{\circ} \mathrm{C}$. Enoate $(124 \mathrm{mg}, 0.50 \mathrm{mmol})$ was introduced via syringe followed by PMHS $(65 \mu \mathrm{L}, 1.0 \mathrm{mmol})$ and $t-\mathrm{BuOH}(52 \mu \mathrm{L}, 0.55 \mathrm{mmol})$. The mixture was stirred at $-30{ }^{\circ} \mathrm{C}$ until complete by TLC $(7.5 \mathrm{~h}$; ether/hexanes $=1 / 9)$. The reaction was quenched with sat. aq. $\mathrm{NaHCO}_{3}$ and diluted with ether. The aqueous layer was extracted with ether and the combined organic layers was washed with brine, dried over anhydrous $\mathrm{MgSO}_{4}$, filtered, and concentrated by rotary evaporation. The residue was purified by flash chromatography (ether/hexanes = $1 / 15)$ to afford the $(S)$-product $(121 \mathrm{mg}, 97 \%)$ as a clear oil. The de was determined by ${ }^{1} \mathrm{H}$ NMR of the corresponding imide derivative to be $24 \%$. Spectroscopic data matched those from experiments above.

Table 1, entry 1c. According to method A, the following amounts were used: $\mathrm{CuCl}(0.99 \mathrm{mg}, 0.01$ $\mathrm{mmol}),(R, S)-\mathrm{PPF}-\mathrm{P}(\mathrm{t}-\mathrm{Bu})_{2}(0.54 \mathrm{mg}, 0.001 \mathrm{mmol}), \mathrm{NaO}-\mathrm{t}-\mathrm{Bu}(0.96 \mathrm{mg}, 0.01 \mathrm{mmol})$, toluene $(1.43 \mathrm{~mL})$, PMHS (130 $\mu \mathrm{L}, 2.0 \mathrm{mmol})$, enoate $Z-1(\mathrm{R}=\mathrm{Me}, \mathrm{R}$ ' = OEt, neat, $248 \mathrm{mg}, 1.0 \mathrm{mmol})$, and $t$-BuOH $(104$ $\mu \mathrm{L}, 1.1 \mathrm{mmol})$. The $(S)$-product ester $(225 \mathrm{mg}, 90 \%)$ was isolated as a clear oil after a reaction time of 
$36 \mathrm{~h}$ at $-20{ }^{\circ} \mathrm{C}$. The de was determined by ${ }^{1} \mathrm{H}$ NMR of the corresponding imide derivative to be $92 \%$. Spectroscopic data matched those from experiments above.

(S)-Ethyl 3-(dimethylphenylsilyl)-3-(phenyl)propanoate (Table 1, entry 2a). According to method A, the following amounts were used: $\mathrm{CuCl}(1.5 \mathrm{mg}, 0.015 \mathrm{mmol}),(R, S)-\mathrm{PPF}-\mathrm{P}(t-\mathrm{Bu})_{2}(2.7 \mathrm{mg}, 0.005$ $\mathrm{mmol}), \mathrm{NaO}-t$-Bu $(1.4 \mathrm{mg}, 0.015 \mathrm{mmol})$, toluene $(1.43 \mathrm{~mL})$, PMHS (65 $\mu \mathrm{L}, 1.0 \mathrm{mmol})$, enoate (155 mg, $0.50 \mathrm{mmol})$, and $t$ - $\mathrm{BuOH}(52 \mu \mathrm{L}, 0.55 \mathrm{mmol})$. The product ester $(139 \mathrm{mg}, 89 \%)$ was isolated as a clear oil after a reaction time of $15 \mathrm{~h}$ at $-30{ }^{\circ} \mathrm{C}$. The de was determined by ${ }^{1} \mathrm{H}$ NMR of the corresponding imide derivative to be $91 \% ; \mathrm{R}_{\mathrm{f}}=0.22$ (ether/hexanes $\left.=1 / 10\right)$, IR (neat): 3069, 2958, 1733, 1600, 1428, 1250, 1164, $1114 \mathrm{~cm}^{-1} ;{ }^{1} \mathrm{H}$ NMR $\left(400 \mathrm{MHz}, \mathrm{CDCl}_{3}\right) \delta 0.22(\mathrm{~s}, 3 \mathrm{H}), 0.26(\mathrm{~s}, 3 \mathrm{H}), 1.04(\mathrm{t}, J=7.1 \mathrm{~Hz}$, $3 \mathrm{H}), 2.63(\mathrm{dd}, J=15.6,4.6 \mathrm{~Hz}, 1 \mathrm{H}), 2.75(\mathrm{dd}, J=15.6,11.3 \mathrm{~Hz}, 1 \mathrm{H}), 2.85(\mathrm{dd}, J=11.3,4.6 \mathrm{~Hz}, 1 \mathrm{H})$, $3.92(\mathrm{~m}, 2 \mathrm{H}), 6.95(\mathrm{~d}, J=7.8 \mathrm{~Hz}, 2 \mathrm{H}), 7.09(\mathrm{t}, J=7.8 \mathrm{~Hz}, 1 \mathrm{H}), 7.20(\mathrm{t}, J=7.8 \mathrm{~Hz}, 2 \mathrm{H}), 7.32-7.43(\mathrm{~m}$, $5 \mathrm{H}) ;{ }^{13} \mathrm{C} \mathrm{NMR}\left(100 \mathrm{MHz}, \mathrm{CDCl}_{3}\right) \delta-5.36,-3.98,14.12,32.42,35.02,60.29,125.03,127.70,127.84$,

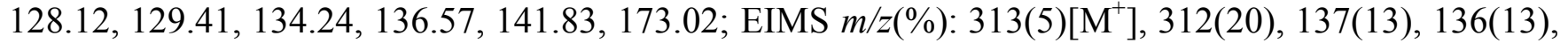
135(100), 105(10), 104(21), 43(10); HREIMS calcd for $\mathrm{C}_{19} \mathrm{H}_{24} \mathrm{O}_{2} \mathrm{Si}$ : 312.1546; found 312.1545[M $\mathrm{M}^{+}$.

Table 1, entry 2b. According to method B, the following amounts were used: $\left(\mathrm{Ph}_{3} \mathrm{P}\right) \mathrm{CuH}(2.7 \mathrm{mg}$, $0.0083 \mathrm{mmol}),(R)$-DTBM-SEGPHOS $(0.98 \mathrm{mg}, 0.00083 \mathrm{mmol})$, toluene $(1.19 \mathrm{~mL})$, enoate $(259 \mathrm{mg}$, $0.83 \mathrm{mmol})$, PMHS (108 $\mu \mathrm{L}, 1.67 \mathrm{mmol})$, and $t$-BuOH $(87 \mu \mathrm{L}, 0.92 \mathrm{mmol})$. The $(S)$-product ester $(236$ $\mathrm{mg}, 90 \%$ ) was isolated as a clear oil after a reaction time of $48 \mathrm{~h}$ at $-20^{\circ} \mathrm{C}$. The de was determined by ${ }^{1} \mathrm{H}$ NMR of the corresponding imide derivative to be $20 \%$. Spectroscopic data matched those from experiments above.

(S)-Octyl 3-(dimethylphenylsilyl)butanoate (Table 1, entry 3a). According to method A, the following amounts were used: $\mathrm{CuCl}(1.5 \mathrm{mg}, 0.015 \mathrm{mmol}),(R, S)-\mathrm{PPF}-\mathrm{P}(t-\mathrm{Bu})_{2}(2.7 \mathrm{mg}, 0.005 \mathrm{mmol})$, $\mathrm{NaO}-t$-Bu $(1.4 \mathrm{mg}, 0.015 \mathrm{mmol})$, toluene $(1 \mathrm{~mL})$, PMHS $(65 \mu \mathrm{L}, 1.0 \mathrm{mmol})$, enoate $(166 \mathrm{mg}, 0.5$ $\mathrm{mmol})$, and $t$-BuOH $(52 \mu \mathrm{L}, 0.55 \mathrm{mmol})$. The product ester $(159 \mathrm{mg}, 95 \%)$ was isolated as a clear oil after a reaction time of $9.5 \mathrm{~h}$ at $-30{ }^{\circ} \mathrm{C}$. The de was determined by ${ }^{1} \mathrm{H}$ NMR of the corresponding imide derivative to be $92 \% ; \mathrm{R}_{\mathrm{f}}=0.31$ (ether/hexanes $=1 / 19$ ); IR (neat): 3070, 2955, 2929, 1734, 1428, 1250, $1208 \mathrm{~cm}^{-1} ;{ }^{1} \mathrm{H}$ NMR $\left(400 \mathrm{MHz}, \mathrm{CDCl}_{3}\right) \delta 0.29(\mathrm{~s}, 6 \mathrm{H}), 0.89(\mathrm{t}, J=6.9 \mathrm{~Hz}, 3 \mathrm{H}), 0.98(\mathrm{~d}, J=7.4 \mathrm{~Hz}, 3 \mathrm{H})$, $1.20-1.38(\mathrm{~m}, 10 \mathrm{H}), 1.45(\mathrm{~m}, 1 \mathrm{H}), 1.59(\mathrm{~m}, 2 \mathrm{H}), 2.05(\mathrm{dd}, J=15.2,11.3 \mathrm{~Hz}, 1 \mathrm{H}), 2.38(\mathrm{dd}, J=15.2,4.0$ $\mathrm{Hz}, 1 \mathrm{H}), 4.02(\mathrm{t}, J=6.8 \mathrm{~Hz}, 2 \mathrm{H}), 7.33-7.40(\mathrm{~m}, 3 \mathrm{H}), 7.48-7.54(\mathrm{~m}, 2 \mathrm{H}) ;{ }^{13} \mathrm{C} \mathrm{NMR}\left(100 \mathrm{MHz}, \mathrm{CDCl}_{3}\right) \delta$ $-5.33,-5.04,14.06,14.45,16.47,22.60,25.90,28.60,29.14,29.16,31.74,36.84,64.43,127.75,129.05$,

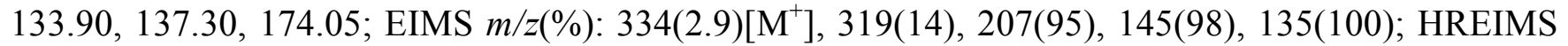
calcd for $\mathrm{C}_{20} \mathrm{H}_{34} \mathrm{O}_{2} \mathrm{Si}$ : 334.2328; found 334.2335[M+].

Table 1, entry 3b. According to method B, the following amounts were used: $\left(\mathrm{Ph}_{3} \mathrm{P}\right) \mathrm{CuH}(4.9 \mathrm{mg}$, $0.015 \mathrm{mmol}),(R)$-DTBM-SEGPHOS $(5.9 \mathrm{mg}, 0.005 \mathrm{mmol})$, toluene $(1 \mathrm{~mL})$, enoate $(166 \mathrm{mg}, 0.50$ $\mathrm{mmol})$, PMHS $(65 \mu \mathrm{L}, 1.0 \mathrm{mmol})$, and $t$-BuOH $(52 \mu \mathrm{L}, 0.55 \mathrm{mmol})$. The $(S)$-product eater $(153 \mathrm{mg}$, $92 \%$ ) was isolated as a clear oil after a reaction time of $7 \mathrm{~h}$ at $-30{ }^{\circ} \mathrm{C}$. The de was determined by ${ }^{1} \mathrm{H}$ NMR of the corresponding imide derivative to be $15 \%$. Spectroscopic data matched those from experiments above.

(R)-Ethyl 3-(dimethylphenylsilyl)butanoate (Table 1 entry 4a). According to method A, the following amounts were used: $\mathrm{CuCl}(1.5 \mathrm{mg}, 0.015 \mathrm{mmol}),(R, S)-\mathrm{PPF}-\mathrm{P}(t-\mathrm{Bu})_{2}(2.7 \mathrm{mg}, 0.005 \mathrm{mmol})$, $\mathrm{NaO}-t$ - $\mathrm{Bu}(1.4 \mathrm{mg}, 0.015 \mathrm{mmol})$, toluene $(1 \mathrm{~mL})$, PMHS $(65 \mu \mathrm{L}, 1.0 \mathrm{mmol})$, enoate $(124 \mathrm{mg}, 0.50$ $\mathrm{mmol})$, and $t$-BuOH $(52 \mu \mathrm{L}, 0.55 \mathrm{mmol})$. The product ester $(123 \mathrm{mg}, 98 \%)$ was isolated as a clear oil after a reaction time of $18 \mathrm{~h}$ at $-78{ }^{\circ} \mathrm{C}$. The de was determined by ${ }^{1} \mathrm{H}$ NMR of the corresponding imide 
derivative to be $>95 \%$. Spectroscopic data matched those from experiments above.

Table 1, entry $4 \mathbf{b}$. According to method A, the following amounts were used: $\mathrm{CuCl}(1.5 \mathrm{mg}, 0.015$ $\mathrm{mmol}),(S, R)$-PPF-P $(t-\mathrm{Bu})_{2}(2.7 \mathrm{mg}, 0.005 \mathrm{mmol}), \mathrm{NaO}-t-\mathrm{Bu}(1.4 \mathrm{mg}, 0.015 \mathrm{mmol})$, toluene $(1 \mathrm{~mL})$, PMHS (65 $\mu \mathrm{L}, 1.0 \mathrm{mmol})$, enoate $(124 \mathrm{mg}, 0.50 \mathrm{mmol})$, and $t$-BuOH $(52 \mu \mathrm{L}, 0.55 \mathrm{mmol})$. The $(S)$ product ester $(119 \mathrm{mg}, 95 \%)$ was isolated as a clear oil after a reaction time of $24 \mathrm{~h}$ at $-78{ }^{\circ} \mathrm{C}$. The de was determined by ${ }^{1} \mathrm{H}$ NMR of the corresponding imide derivative to be $>95 \%$. Spectroscopic data matched those from experiments above.

Table 1, entry 4c. According to method B, the following amounts were used: $\left(\mathrm{Ph}_{3} \mathrm{P}\right) \mathrm{CuH}(4.9 \mathrm{mg}$, $0.015 \mathrm{mmol}),(R)$-DTBM-SEGPHOS $(5.9 \mathrm{mg}, 0.005 \mathrm{mmol})$, toluene $(1 \mathrm{~mL})$, enoate $(124 \mathrm{mg}, 0.50$ $\mathrm{mmol})$, PMHS (65 $\mu \mathrm{L}, 1.0 \mathrm{mmol})$, and $t-\mathrm{BuOH}(52 \mu \mathrm{L}, 0.55 \mathrm{mmol})$. The $(R)$-product ester $(122 \mathrm{mg}$, $98 \%$ ) was isolated as a clear oil after a reaction time of $5 \mathrm{~h}$ at $-30{ }^{\circ} \mathrm{C}$. The de was determined by ${ }^{1} \mathrm{H}$ NMR of the corresponding imide derivative to be $83 \%$. Spectroscopic data matched those from experiments above.

(R)-Methyl 3-(dimethylphenylsilyl)butanoate (Table 1, entry 5). According to method A, the following amounts were used: $\mathrm{CuCl}(1.5 \mathrm{mg}, 0.015 \mathrm{mmol}),(R, S)-\mathrm{PPF}-\mathrm{P}(t-\mathrm{Bu})_{2}(2.7 \mathrm{mg}, 0.005 \mathrm{mmol})$, $\mathrm{NaO}-t$-Bu $(1.4 \mathrm{mg}, 0.015 \mathrm{mmol})$, toluene $(1 \mathrm{~mL})$, PMHS $(65 \mu \mathrm{L}, 1.0 \mathrm{mmol})$, enoate $(117 \mathrm{mg}, 0.50$ $\mathrm{mmol})$, and $t \mathrm{BuOH}(52 \mu \mathrm{L}, 0.55 \mathrm{mmol})$. The product ester $(115 \mathrm{mg}, 97 \%)$ was isolated as a clear oil after a reaction time of $12 \mathrm{~h}$ at $-60{ }^{\circ} \mathrm{C}$. The de was determined by ${ }^{1} \mathrm{H}$ NMR of the corresponding imide derivative to be $>95 \% ; R_{\mathrm{f}}=0.32$ (ethyl acetate/hexanes $=1 / 20$ ); IR (neat): $3071,2953,2876,1738$, $1428,1251,1211,1153,1111,1005 \mathrm{~cm}^{-1} ;{ }^{1} \mathrm{H}$ NMR $\left(400 \mathrm{MHz}, \mathrm{CDCl}_{3}\right) \delta 0.29(\mathrm{~s}, 6 \mathrm{H}), 0.98$ (d, $J=7.4$ $\mathrm{Hz}, 3 \mathrm{H}), 1.45(\mathrm{~m}, 1 \mathrm{H}), 2.08(\mathrm{dd}, J=15.2,11.4 \mathrm{~Hz}, 1 \mathrm{H}), 2.41(\mathrm{dd}, J=15.2,4.0 \mathrm{~Hz}, 1 \mathrm{H}), 3.63(\mathrm{~s}, 3 \mathrm{H})$, 7.35-7.39 (m, 3H), 7.48-7.53 (m, 2H); ${ }^{13} \mathrm{C}$ NMR $\left(100 \mathrm{MHz}, \mathrm{CDCl}_{3}\right) \delta-5.19,-4.82,14.70,16.70,36.83$, 51.61, 127.98, 129.30, 134.10, 137.43, 174.61; EIMS $m / z(\%): 236(10)\left[\mathrm{M}^{+}\right], 221(29), 159(13), 151(23)$, 145(12), 136(13), 135(100), 105(10), 89(12), 42(10); HREIMS calcd for $\mathrm{C}_{13} \mathrm{H}_{20} \mathrm{O}_{2} \mathrm{Si}$ : 236.1232; found $236.1232\left[\mathrm{M}^{+}\right]$.

(R)-Methyl 6-(4-methoxybenzyloxy)-3-(dimethylphenylsilyl)hexanoate (Table 1, entry 6). According to method $\mathrm{A}$, the following amounts were used: $\mathrm{CuCl}(1.5 \mathrm{mg}, 0.015 \mathrm{mmol}),(R, S)$-PPF-P $(t-$ $\mathrm{Bu})_{2}(2.7 \mathrm{mg}, 0.005 \mathrm{mmol}), \mathrm{NaO}-t-\mathrm{Bu}(1.4 \mathrm{mg}, 0.015 \mathrm{mmol})$, toluene $(1 \mathrm{~mL})$, PMHS $(65 \mu \mathrm{L}, 1.0$ $\mathrm{mmol})$, enoate $(199 \mathrm{mg}, 0.50 \mathrm{mmol})$, and $t \mathrm{BuOH}(52 \mu \mathrm{L}, 0.55 \mathrm{mmol})$. The product ester $(166 \mathrm{mg}$, $83 \%$ ) was isolated as a clear oil after a reaction time of $24 \mathrm{~h}$ at $-30{ }^{\circ} \mathrm{C}$. The de was determined by ${ }^{1} \mathrm{H}$ NMR of the corresponding imide derivative to be $>95 \% ; R_{\mathrm{f}}=0.25$ (ethyl acetate/hexanes $=1 / 10$ ); IR (neat): 3071, 3000, 2950, 2854, 1736, 1613, $1512 \mathrm{~cm}^{-1} ;{ }^{1} \mathrm{H}$ NMR (400 MHz, $\left.\mathrm{CDCl}_{3}\right) \delta 0.30(\mathrm{~s}, 3 \mathrm{H}), 0.31$ (s, $3 \mathrm{H}), 1.26-1.68(\mathrm{~m}, 5 \mathrm{H}), 2.25(\mathrm{dd}, J=15.6,8.1 \mathrm{~Hz}, 1 \mathrm{H}), 2.36(\mathrm{dd}, J=15.6,5.7 \mathrm{~Hz}, 1 \mathrm{H}), 3.37(\mathrm{t}, J=$ $6.3 \mathrm{~Hz}, 2 \mathrm{H}), 3.57(\mathrm{~s}, 3 \mathrm{H}), 3.81(\mathrm{~s}, 3 \mathrm{H}), 4.38(\mathrm{~s}, 2 \mathrm{H}), 6.88(\mathrm{~d}, J=8.7 \mathrm{~Hz}, 2 \mathrm{H}), 7.24(\mathrm{~d}, J=8.6 \mathrm{~Hz}, 2 \mathrm{H})$, 7.33-7.39 (m, 3H), 7.49-7.53 (m, 2H); ${ }^{13} \mathrm{C}$ NMR (100 MHz, $\left.\mathrm{CDCl}_{3}\right) \quad \delta-4.23,-3.94,21.8,26.8,29.0$, $34.8,51.6,55.4,70.1,72.5,113.8,127.9,129.2,129.3,130.8,134.1,137.9,159.2,174.8$; EIMS $m / z(\%)$ : 400(1)[ $\left.\mathrm{M}^{+}\right], 279(6), 221(10), 135(60), 121(100), 77(5)$; HRESIMS calcd for $\mathrm{C}_{23} \mathrm{H}_{32} \mathrm{NaO}_{4} \mathrm{Si}: 423.1968$; found $423.1975\left[\mathrm{M}^{+}+\mathrm{Na}\right]$.

(R)-Methyl 3-(dimethylphenylsilyl)-3-(3,4,5-trimethoxyphenyl)propanoate (Table 1, entry 7). According to method $\mathrm{A}$, the following amount were used: $\mathrm{CuCl}(1.5 \mathrm{mg}, 0.015 \mathrm{mmol}),(R, S)-\mathrm{PPF}-\mathrm{P}(t-$ $\mathrm{Bu})_{2}(2.7 \mathrm{mg}, 0.005 \mathrm{mmol}), \mathrm{NaO}-t-\mathrm{Bu}(1.4 \mathrm{mg}, 0.015 \mathrm{mmol})$, toluene $(0.5 \mathrm{~mL})$, PMHS $(65 \mu \mathrm{L}, 1.0$ $\mathrm{mmol})$, enoate $(120 \mathrm{mg}, 0.31 \mathrm{mmol})$, and $t-\mathrm{BuOH}(52 \mu \mathrm{L}, 0.55 \mathrm{mmol})$. The product ester $(118 \mathrm{mg}$, $98 \%$ ) was isolated as a clear oil after a reaction time of $24 \mathrm{~h}$ at $0{ }^{\circ} \mathrm{C} ; \mathrm{R}_{\mathrm{f}}=0.30$ (ethyl acetate/hexanes $=$ 
3/7). The ee was determined on a Daicel Chiralpak OD-H HPLC column with hexane : 2-propanol = $100: 5$, flow $=5.0 \mathrm{~mL} / \mathrm{min}$. Retention times: $19.6 \mathrm{~min}[(R)$-enantiomer], $21.4 \mathrm{~min}[(S)$-enantiomer]; 81.6\% ee; IR (neat): 2953, 1736, 1586, 1455, $1245 \mathrm{~cm}^{-1} ;{ }^{1} \mathrm{H}$ NMR $\left(400 \mathrm{MHz}, \mathrm{CDCl}_{3}\right) \delta 0.25$ (s, 3H), $0.26(\mathrm{~s}, 3 \mathrm{H}), 2.67-2.78(\mathrm{~m}, 3 \mathrm{H}), 3.53(\mathrm{~s}, 3 \mathrm{H}), 3.68(\mathrm{~s}, 6 \mathrm{H}), 3.80(\mathrm{~s}, 3 \mathrm{H}), 6.04(\mathrm{~s}, 2 \mathrm{H}), 7.31-7.39(\mathrm{~m}, 5 \mathrm{H})$; ${ }^{13} \mathrm{C}$ NMR $\left(100 \mathrm{MHz}, \mathrm{CDCl}_{3}\right) \delta-4.83,-4.20,32.74,34.86,51.68,55.98,61.06,104.54,127.89,129.56$, 134.44, 135.42, 136.55, 137.55, 152.83, 173.68; EIMS $m / z(\%): 388(16)\left[\mathrm{M}^{+}\right], 387(36), 373(20), 372(46)$, 330(18), 194(10), 193(37), 178(12), 135(15), 134(100); HREIMS calcd for $\mathrm{C}_{21} \mathrm{H}_{28} \mathrm{O}_{5} \mathrm{Si}: 388.1706$; found $388.1711\left[\mathrm{M}^{+}\right]$.

(R)-Methyl 3-(dimethylphenylsilyl)-3-(4-chlorophenyl)propanoate (Table 1, entry 8). According to method $\mathrm{A}$, the following amounts were used: $\mathrm{CuCl}(1.5 \mathrm{mg}, 0.015 \mathrm{mmol}),(R, S)-\mathrm{PPF}-\mathrm{P}(t-\mathrm{Bu})_{2}(2.7 \mathrm{mg}$, $0.005 \mathrm{mmol}), \mathrm{NaO}-t$-Bu $(1.4 \mathrm{mg}, 0.015 \mathrm{mmol})$, toluene $(0.5 \mathrm{~mL})$, PMHS $(65 \mu \mathrm{L}, 1.0 \mathrm{mmol})$, enoate $(100 \mathrm{mg}, 0.3 \mathrm{mmol})$, and $t$-BuOH $(52 \mu \mathrm{L}, 0.55 \mathrm{mmol})$. The product ester $(82 \mathrm{mg}, 82 \%)$ was isolated as a clear oil after a reaction time of $24 \mathrm{~h}$ at $-30{ }^{\circ} \mathrm{C} . \mathrm{R}_{\mathrm{f}}=0.40\left(\mathrm{CH}_{2} \mathrm{Cl}_{2} /\right.$ hexanes $\left.=1 / 1\right)$. The ee was determined on a Daicel Chiralpak OD-H HPLC column with hexane : 2-propanol = $100: 1$, flow $=5.0$ $\mathrm{mL} / \mathrm{min}$. Retention times: $20.2 \mathrm{~min}$ [(R)-enantiomer], $21.5 \mathrm{~min}$ [(S)-enantiomer]; 97.3\% ee; IR (neat): 3069, 2953, 1737, 1428, $1250 \mathrm{~cm}^{-1}$; ${ }^{1} \mathrm{H}$ NMR (400 MHz, $\left.\mathrm{CDCl}_{3}\right) \delta 0.23(\mathrm{~s}, 3 \mathrm{H}), 0.25(\mathrm{~s}, 3 \mathrm{H}), 2.61-2.85$ $(\mathrm{m}, 3 \mathrm{H}), 3.49(\mathrm{~s}, 3 \mathrm{H}), 6.86(\mathrm{~d}, J=8.0 \mathrm{~Hz}, 2 \mathrm{H}), 7.16(\mathrm{~d}, J=8.0 \mathrm{~Hz}, 2 \mathrm{H}) \quad 7.34-7.40(\mathrm{~m}, 5 \mathrm{H}) ;{ }^{13} \mathrm{C}$ NMR $\left(100 \mathrm{MHz}, \mathrm{CDCl}_{3}\right) \delta-5.29,-4.07,32.08,34.77,51.83,128.06,128.44,128.94,129.73,130.78,134.33$,

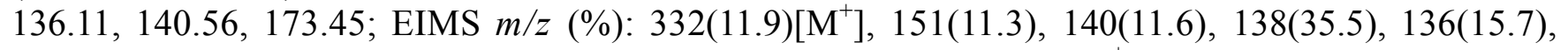
135(100); HREIMS calcd for $\mathrm{C}_{18} \mathrm{H}_{21} \mathrm{O}_{2} \mathrm{SiCl}$ : 332.0999; found 332.0996[ $\left.\mathrm{M}^{+}\right]$.

(R)-Ethyl 3-(dimethylphenylsilyl)-4-(phenyl)butanoate (Table 1, entry 9). According to method A, the following amount were used: $\mathrm{CuCl}(1.5 \mathrm{mg}, 0.015 \mathrm{mmol}),(R, S)-\mathrm{PPF}-\mathrm{P}(t-\mathrm{Bu})_{2}(2.7 \mathrm{mg}, 0.005$ $\mathrm{mmol}), \mathrm{NaO}-t-\mathrm{Bu}(1.4 \mathrm{mg}, 0.015 \mathrm{mmol})$, toluene $(0.5 \mathrm{~mL})$, PMHS $(65 \mu \mathrm{L}, 1.0 \mathrm{mmol})$, enoate $(100 \mathrm{mg}$, $0.31 \mathrm{mmol})$, and $t$-BuOH $(52 \mu \mathrm{L}, 0.55 \mathrm{mmol})$. The product ester $(76 \mathrm{mg}, 76 \%)$ was isolated as a clear oil after a reaction time of $24 \mathrm{~h}$ at $0{ }^{\circ} \mathrm{C} . \mathrm{R}_{\mathrm{f}}=0.40($ ether/hexanes $=1 / 9)$. The ee was determined on a Daicel Chiralpak OD-H HPLC column with hexane : 2-propanol $=100: 1$, flow $=5.0 \mathrm{~mL} / \mathrm{min}$. Retention times: $6.2 \min [(R)$-enantiomer], $7.1 \min [(S)$-enantiomer]; 98.5\% ee. IR (neat): 2955, 1731, $1245 \mathrm{~cm}^{-1} ;{ }^{1} \mathrm{H}$ NMR $\left(400 \mathrm{MHz}, \mathrm{CDCl}_{3}\right) \delta 0.29(\mathrm{~s}, 3 \mathrm{H}), 0.30(\mathrm{~s}, 3 \mathrm{H}), 1.15(\mathrm{t}, J=6.8 \mathrm{~Hz}, 3 \mathrm{H}), 1.78-1.85$ $(\mathrm{m}, 1 \mathrm{H}), 2.19-2.5(\mathrm{~m}, 2 \mathrm{H}), 2.75(\mathrm{dd}, J=10.4,14 \mathrm{~Hz}, 1 \mathrm{H}), 2.83(\mathrm{dd}, J=8.8,4.8 \mathrm{~Hz}, 1 \mathrm{H}), 3.86(\mathrm{q}, J=7.2$ $\mathrm{Hz}, 2 \mathrm{H}), 7.14-7.27(\mathrm{~m}, 5 \mathrm{H}), 7.37-7.39(\mathrm{~m}, 3 \mathrm{H}), 7.53-7.55(\mathrm{~m}, 2 \mathrm{H}) ;{ }^{13} \mathrm{C} \mathrm{NMR}\left(100 \mathrm{MHz}, \mathrm{CDCl}_{3}\right) \delta-$ $4.24,-4.05,14.31,24.31,34.68,36.35,60.40,126.17,128.03,129.25,129.35,137.71,141.51,173.79$; EIMS $m / z(\%):$ 249(21), 235(26), 137(24), 135(100), 129(13), 117(23), 105(13), 91(24), 75(15); HREIMS calcd for $\mathrm{C}_{19} \mathrm{H}_{23} \mathrm{O}_{2} \mathrm{Si}$ : 311.1467; found 311.1477 [ $\left.\mathrm{M}^{+}-\mathrm{CH}_{3}\right]$.

\section{Substrates.}

General procedure for synthesis of morpholine amides from acid chlorides. ${ }^{1}$ The acid chloride (1 equiv) was added to a flask containing $\mathrm{CH}_{2} \mathrm{Cl}_{2}$ (enough to make a $1.0 \mathrm{M}$ solution of the acid chloride). The flask was cooled to $0{ }^{\circ} \mathrm{C}$, and morpholine ( 3 equiv) was added slowly. The reaction was allowed to warm to rt and stirred for $30 \mathrm{~min}$ at which time it was diluted with EtOAc, then washed with $1 \mathrm{M}$ hydrochloric acid, saturated sodium bicarbonate, and brine. The organic layer was dried over anhydrous $\mathrm{Na}_{2} \mathrm{SO}_{4}$, filtered, and concentrated in vacuo. The resulting imides were used without further purification.

General procedure for synthesis of acylsilanes from morpholine amides. ${ }^{1}$ The morpholine amide $(2$ mmol) was added to a flame-dried $50 \mathrm{~mL}$ round-bottomed flask under dry nitrogen. To this amide, THF $(3 \mathrm{~mL})$ was added, and the solution was then cooled to $-78{ }^{\circ} \mathrm{C}$. Dimethylphenylsilyllithium $(1.0 \mathrm{M}$ 
solution in THF, $3 \mathrm{~mL}$ ) was added dropwise via syringe, and the reaction was allowed to stir for 1.5 hours, after which time it was quenched at $-78{ }^{\circ} \mathrm{C}$ (to prevent decomposition) by the addition of $4 \mathrm{~mL}$ of saturated aqueous ammonium chloride. The resulting heterogeneous mixture was allowed to warm to $\mathrm{rt}$ and then stirred for an additional $30 \mathrm{~min}$. The reaction was then partitioned between water $(5 \mathrm{~mL})$ and ether $(5 \mathrm{~mL})$, the phases were separated, and the aqueous phase was extracted with ether $(2 \times 5 \mathrm{~mL})$. The combined organics were washed with water $(2 \times 20 \mathrm{~mL})$ and once with brine $(20 \mathrm{~mL})$, dried over anhydrous $\mathrm{MgSO}_{4}$, filtered, and concentrated in vacuo. The residue was purified by flash column chromatography using silica gel yielding pure acylsilane.

General HWE procedure for syntheses of $(\boldsymbol{E})-\boldsymbol{\beta}$-silyl enoates from acylsilanes. ${ }^{1} \quad$ To a mineral oil dispersion of sodium hydride $(2 \mathrm{mmol})$ was added a THF $(2 \mathrm{~mL})$ solution of triethylphosphonoacetate $(2.0 \mathrm{mmol})$ at $\mathrm{rt}$ with stirring. After $30 \mathrm{~min}$, acylsilane $(2.0 \mathrm{mmol})$ was added dropwise and the reaction mixture was stirred overnight. The reaction mixture was quenched with DI water $(5 \mathrm{~mL})$ and diluted with ether $(5 \mathrm{~mL})$. The aqueous phase was further extracted with ether $(2 \times 5 \mathrm{~mL})$. The combined organics were washed with $\mathrm{NaHCO}_{3}(2 \times 20 \mathrm{~mL})$, once with brine $(20 \mathrm{~mL})$, and then further dried over anhydrous $\mathrm{MgSO}_{4}$, filtered, and concentrated in vacuo. The residue, which consisted of both $(E)$ and $(Z)$ isomers, was purified by flash column chromatography using silica gel yielding pure $(E)$ eneoate.

General Peterson olefination procedure for syntheses of $(\boldsymbol{Z})$ - $\boldsymbol{\beta}$-silyl enoates from acylsilanes. ${ }^{1}$ To a THF (3 mL) solution of 1,1,1,3,3,3-hexamethyldisilazane (194 mg, $1.2 \mathrm{mmol}$ ) was added a hexane solution of $n$-butyllithium $(1.6 \mathrm{M}, 0.75 \mathrm{~mL}, 1.2 \mathrm{mmol})$ at $0{ }^{\circ} \mathrm{C}$ and the reaction mixture was stirred for 20 min. After cooling to $-78^{\circ} \mathrm{C}$, ethyl (trimethylsilyl)acetate $(192 \mathrm{mg}, 1.2 \mathrm{mmol})$ in $\mathrm{THF}(1 \mathrm{~mL}) \mathrm{was}$ added, and stirring was continued for $15 \mathrm{~min}$. A THF $(1 \mathrm{~mL})$ solution of acylsilane $(1 \mathrm{mmol})$ was added, and the reaction mixture was stirred at the same temperature for $1 \mathrm{~h}$. The cooling bath was removed, and the reaction mixture was allowed to warm to $\mathrm{rt}$. A saturated $\mathrm{NH}_{4} \mathrm{Cl}$ aqueous solution (10 $\mathrm{mL}$ ) was added, and the organic materials were extracted with ether $(2 \times 15 \mathrm{~mL})$, dried over anhydrous $\mathrm{Na}_{2} \mathrm{SO}_{4}$, and concentrated under reduced pressure. The residue, which consisted of both $(E)$ and $(Z)$ isomers, was purified by flash column chromatography using silica gel yielding pure $(Z)$ enoate.

Ethyl (Z)-3-(dimethylphenylsilyl)-2-butenoate (Table 1, entry 1). Purified by $\mathrm{SiO}_{2}$ chromatography (ether/hexanes =1/24); $\mathrm{R}_{\mathrm{f}}=0.56$ (ether/hexanes = 1/9); IR (neat): 3069, 2955, 1717, 1605, 1429, 1330, 1247, 1200, 1112, $1042 \mathrm{~cm}^{-1} ;{ }^{1} \mathrm{H}$ NMR (400 MHz, $\left.\mathrm{CDCl}_{3}\right) \delta 0.48$ (s, 6H), 1.15 (t, J=7.1 Hz, 3H), 1.96 $(\mathrm{d}, J=1.8 \mathrm{~Hz}, 3 \mathrm{H}), 4.01(\mathrm{q}, J=7.1 \mathrm{~Hz}, 2 \mathrm{H}), 6.43(\mathrm{q}, J=1.8 \mathrm{~Hz}, 1 \mathrm{H}), 7.30-7.38(\mathrm{~m}, 3 \mathrm{H}), 7.49-7.56(\mathrm{~m}$, $2 \mathrm{H}) ;{ }^{13} \mathrm{C}$ NMR $\left(100 \mathrm{MHz}, \mathrm{CDCl}_{3}\right) \delta-1.88,14.22,26.97,60.02,127.68,128.76,132.19,133.77,138.86$, 159.42, 166.41; EIMS m/z(\%): 233(38) [ $\left.\mathrm{M}^{+}-\mathrm{CH}_{3}\right], 205(16), 203(12), 172(14), 171(100)$; HREIMS calcd for $\mathrm{C}_{13} \mathrm{H}_{17} \mathrm{O}_{2} \mathrm{Si}$ : 233.0998; found 233.0993[M $\left.\mathrm{M}^{+}-\mathrm{CH}_{3}\right]$.

Ethyl (Z)-3-(dimethylphenylsilyl)-3-phenyl-2-propenoate (Table 1, entry 2). Purified by $\mathrm{SiO}_{2}$ chromatography (ether/hexanes $=1 / 15) ; \mathrm{R}_{\mathrm{f}}=0.45$ (ether/ hexanes $=1 / 10$ ); IR (neat): 3069, 2979, 1719, 1591, 1321, 1182, 1112, $1032 \mathrm{~cm}^{-1} ;{ }^{1} \mathrm{H}$ NMR (400 MHz, $\left.\mathrm{CDCl}_{3}\right) \delta 0.39(\mathrm{~s}, 6 \mathrm{H}), 1.14(\mathrm{t}, J=7.1 \mathrm{~Hz}, 3 \mathrm{H})$, $4.00(\mathrm{q}, J=7.1 \mathrm{~Hz}, 2 \mathrm{H}), 6.49(\mathrm{~s}, 1 \mathrm{H}), 7.06-7.11(\mathrm{~m}, 2 \mathrm{H}), 7.20-7.34(\mathrm{~m}, 6 \mathrm{H}), 7.53-7.59(\mathrm{~m}, 2 \mathrm{H}) ;{ }^{13} \mathrm{C}$ $\operatorname{NMR}\left(100 \mathrm{MHz}, \mathrm{CDCl}_{3}\right) \delta-0.50,14.21,60.55,126.74,126.89,127.70,128.14,128.83,133.87,134.64$, 138.61, 145.11, 162.27, 166.62; EIMS m/z(\%): 310(0.9)[ $\left.\mathrm{M}^{+}\right], 295(89), 267(99), 233(100)$; HREIMS calcd for $\mathrm{C}_{19} \mathrm{H}_{22} \mathrm{O}_{2} \mathrm{Si}: 310.4623$; found $310.1399\left[\mathrm{M}^{+}\right]$.

Octyl (Z)-3-(dimethylphenylsilyl)-2-butenoate (Table 1, entry 3). Purified by $\mathrm{SiO}_{2}$ chromatography (ether/hexanes =1/50); $\mathrm{R}_{\mathrm{f}}=0.52$ (ether/hexanes =1/19); IR (neat): 3069, 2928, 1717, 1604, 1331, 1246, $1197 \mathrm{~cm}^{-1} ;{ }^{1} \mathrm{H}$ NMR $\left(400 \mathrm{MHz}, \mathrm{CDCl}_{3}\right) \delta 0.48(\mathrm{~s}, 6 \mathrm{H}), 0.89(\mathrm{t}, J=6.9 \mathrm{~Hz}, 3 \mathrm{H}), 1.20-1.35(\mathrm{~m}, 10 \mathrm{H})$, 
$1.52(\mathrm{~m}, 2 \mathrm{H}), 1.96(\mathrm{~d}, J=1.7 \mathrm{~Hz}, 3 \mathrm{H}), 3.94(\mathrm{t}, J=6.8 \mathrm{~Hz}, 2 \mathrm{H}), 6.43(\mathrm{q}, J=1.7 \mathrm{~Hz}, 1 \mathrm{H}), 7.29-7.37(\mathrm{~m}$, $3 \mathrm{H}), 7.49-7.56(\mathrm{~m}, 2 \mathrm{H}) ;{ }^{13} \mathrm{C} \mathrm{NMR}\left(100 \mathrm{MHz}, \mathrm{CDCl}_{3}\right) \delta-1.87,14.25,22.81,26.04,27.01,28.74,29.34$, $29.37,31.96,64.37,127.72,128.81,132.12,133.85,138.93,159.50,166.62$; EIMS $\mathrm{m} / z(\%)$ : 317(33)[ $\left.\mathrm{M}^{+}-\mathrm{CH}_{3}\right], 256(20), 255(100)$; HREIMS calcd for $\mathrm{C}_{19} \mathrm{H}_{29} \mathrm{O}_{2} \mathrm{Si}: 317.1937$; found 317.1934[ $\mathrm{M}^{+}-$ $\left.\mathrm{CH}_{3}\right]$.

Ethyl (E)-3-(dimethylphenylsilyl)-2-butenoate (Table 1 entry 4). Purified by $\mathrm{SiO}_{2}$ chromatography $($ ether/hexanes $=1 / 24) ; \mathrm{R}_{\mathrm{f}}=0.43$ (ether/hexanes = 1/9); IR (neat): 3070, 2958, 1716, 1610, 1428, 1337, 1250, 1183, 1113, $1038 \mathrm{~cm}^{-1}$; ${ }^{1} \mathrm{H}$ NMR (400 MHz, $\left.\mathrm{CDCl}_{3}\right) \delta 0.42(\mathrm{~s}, 6 \mathrm{H}), 1.30(\mathrm{t}, J=7.2 \mathrm{~Hz}, 3 \mathrm{H}), 2.19$ $(\mathrm{d}, J=1.8 \mathrm{~Hz}, 3 \mathrm{H}), 4.17(\mathrm{q}, J=7.2 \mathrm{~Hz}, 2 \mathrm{H}), 6.10(\mathrm{q}, J=1.8 \mathrm{~Hz}, 1 \mathrm{H}), 7.33-7.43(\mathrm{~m}, 3 \mathrm{H}), 7.47-7.54(\mathrm{~m}$, $2 \mathrm{H}) ;{ }^{13} \mathrm{C} \mathrm{NMR}\left(100 \mathrm{MHz}, \mathrm{CDCl}_{3}\right) \delta-3.84,14.45,17.62,59.85,127.94,128.11,129.60,134.14,136.28$, 160.01, 165.76. CIMS $m / z(\%): 249(100)[\mathrm{M}+\mathrm{H}]^{+}$, 203(32), 170(57); HRCIMS calcd for $\mathrm{C}_{14} \mathrm{H}_{21} \mathrm{O}_{2} \mathrm{Si}_{\text {: }}$ 249.1311; found 249.1308[M+H] .

Methyl (E)-3-(dimethylphenylsilyl)-2-butenoate (Table 1, entry 5). Purified by $\mathrm{SiO}_{2}$ chromatography (ethyl acetate/hexanes $=1 / 50) ; \mathrm{R}_{\mathrm{f}}=0.36($ ethyl acetate/hexanes $=1 / 20) ;{ }^{1} \mathrm{H} \mathrm{NMR}\left(400 \mathrm{MHz}, \mathrm{CDCl}_{3}\right) \delta$ $0.41(\mathrm{~s}, 6 \mathrm{H}), 2.18(\mathrm{~d}, J=2.0 \mathrm{~Hz}, 3 \mathrm{H}), 3.69(\mathrm{~s}, 3 \mathrm{H}), 6.08(\mathrm{q}, J=2.0 \mathrm{~Hz}, 1 \mathrm{H}), 7.35-7.38(\mathrm{~m}, 3 \mathrm{H}), 7.47-$ $7.49(\mathrm{~m}, 2 \mathrm{H}) ;{ }^{13} \mathrm{C}$ NMR $\left(100 \mathrm{MHz}, \mathrm{CDCl}_{3}\right) \delta-3.89,17.62,51.0,127.43,128.09,129.61,134.10$, 136.14, 160.56, 166.06; HREIMS calcd for $\mathrm{C}_{12} \mathrm{H}_{15} \mathrm{O}_{2} \mathrm{Si}$ : 219.1481; found 219.1846[M- $\left.\mathrm{CH}_{3}\right]^{+}$.

Methyl (E)-6-(4-methoxybenzyloxy)-3-(dimethylphenylsilyl)-2-hexenoate (Table 1, entry 6). Purified by $\mathrm{SiO}_{2}$ chromatography (ethyl acetate/hexanes $\left.=1 / 20\right) ; \mathrm{R}_{\mathrm{f}}=0.29$ (ethyl acetate/hexanes $=$ 1/10); ${ }^{1} \mathrm{H}$ NMR $\left(400 \mathrm{MHz}, \mathrm{CDCl}_{3}\right) \delta 0.43(\mathrm{~s}, 6 \mathrm{H}), 1.63(\mathrm{~m}, 2 \mathrm{H}), 2.69(\mathrm{t}, J=8.0 \mathrm{~Hz}, 2 \mathrm{H}), 3.41(\mathrm{t}, J=6.7$ $\mathrm{Hz}, 2 \mathrm{H}), 3.68(\mathrm{~s}, 3 \mathrm{H}), 3.81(\mathrm{~s}, 3 \mathrm{H}), 4.37(\mathrm{~s}, 2 \mathrm{H}), 6.09(\mathrm{~s}, 1 \mathrm{H}), 6.87(\mathrm{~d}, J=8.7 \mathrm{~Hz}, 2 \mathrm{H}), 7.23(\mathrm{~d}, J=8.7$ $\mathrm{Hz}, 2 \mathrm{H}), 7.34-7.40(\mathrm{~m}, 3 \mathrm{H}), 7.49-7.52(\mathrm{~m}, 2 \mathrm{H}) ;{ }^{13} \mathrm{C}$ NMR $\left(100 \mathrm{MHz}, \mathrm{CDCl}_{3}\right) \delta-3.21,28.27,29.71$, 51.09, 55.34, 70.29, 72.32, 113.77, 127.96, 128.07, 129.25, 129.58, 130.88, 134.15, 136.45, 159.12, 164.72, 165.63; HREIMS calcd for $\mathrm{C}_{23} \mathrm{H}_{30} \mathrm{O}_{4} \mathrm{Si}$ : 398.1913; found 398.1920[M] $]^{+}$

Methyl (E)-3-(3,4,5-trimethoxyphenyl)-3-(dimethylphenylsilyl)acrylate (Table 1, entry 7). Purified by $\mathrm{SiO}_{2}$ chromatography (ethyl acetate/hexanes $\left.=1 / 4\right) ; \mathrm{R}_{\mathrm{f}}=0.4$ (ethyl acetate/hexanes $=3 / 7$ ); IR (neat): 2952, 1732, 1578, 1500, 1238, $1127 \mathrm{~cm}^{-1} ;{ }^{1} \mathrm{H}$ NMR $\left(400 \mathrm{MHz}, \mathrm{CDCl}_{3}\right) \delta 0.40(\mathrm{~s}, 6 \mathrm{H}), 3.55(\mathrm{~s}$, $3 \mathrm{H}), 3.63(\mathrm{~s}, 6 \mathrm{H}), 3.82(\mathrm{~s}, 3 \mathrm{H}), 5.97(\mathrm{~s}, 2 \mathrm{H}), 6.25(\mathrm{~s}, 1 \mathrm{H}), 7.33-7.39(\mathrm{~m}, 3 \mathrm{H}), 7.48(\mathrm{~d}, J=2.0 \mathrm{~Hz}, 2 \mathrm{H})$; ${ }^{13} \mathrm{C}$ NMR $\left(100 \mathrm{MHz}, \mathrm{CDCl}_{3}\right) \delta-3.59,51.47,55.95,61.08,103.44,128.11,128.63,129.80,134.46$, 136.03, 136.28, 136.39, 152.77, 161.25, 165.96; EIMS m/z(\%): 386(50)[M $\left.{ }^{+}\right], 372(31), 371(73), 355(23)$, 310(39), 309(94), 135(100); HREIMS calcd for $\mathrm{C}_{21} \mathrm{H}_{26} \mathrm{O}_{5} \mathrm{Si}$ : 386.1550; found 386.5554 [M ${ }^{+}$.

Methyl (E)-3-(4-chlorophenyl)-3-(dimethylphenylsilyl)acrylate (Table 1 entry 8). Purified by $\mathrm{SiO}_{2}$ chromatography $\left(\mathrm{CH}_{2} \mathrm{Cl}_{2} /\right.$ hexanes $\left.=1 / 3\right) ; \mathrm{R}_{\mathrm{f}}=0.5\left(\mathrm{CH}_{2} \mathrm{Cl}_{2} /\right.$ hexanes $\left.=1 / 1\right) ; \mathrm{IR}$ (neat): 2950, 1725, 1590, 1500, 1486, 1176, $831 \mathrm{~cm}^{-1}$; ${ }^{1} \mathrm{H}$ NMR $\left(400 \mathrm{MHz}, \mathrm{CDCl}_{3}\right) \delta 0.39(\mathrm{~s}, 6 \mathrm{H}), 3.54(\mathrm{~s}, 3 \mathrm{H}), 6.45(\mathrm{~s}, 1 \mathrm{H}), 6.99$ $(\mathrm{d}, J=2.0 \mathrm{~Hz}, 2 \mathrm{H}), 7.24(\mathrm{~d}, J=2.0 \mathrm{~Hz}, 2 \mathrm{H}), 3.28(\mathrm{dd}, J=0.8,2.0 \mathrm{~Hz}, 3 \mathrm{H}), 7.50-7.53(\mathrm{~m}, 2 \mathrm{H}) ;{ }^{13} \mathrm{C}$ NMR $\left(100 \mathrm{MHz}, \mathrm{CDCl}_{3}\right) \delta-0.68,51.62,127.83,128.13,128.37,129.04,132.95,133.81,134.47$, 138.10, 143.47, 161.53, 166.79; EIMS $m / z(\%): 314(28), 254(39), 253(19), 252(100), 134(21), 78(38)$; HREIMS calcd for $\mathrm{C}_{12} \mathrm{H}_{14} \mathrm{ClO}_{2} \mathrm{Si}$ : 253.0452; found $253.0443\left[\mathrm{M}^{+}-\mathrm{C}_{6} \mathrm{H}_{6}\right]$.

Ethyl (E)-3-(dimethylphenylsilyl)-4-(phenyl)-2-butenoate (Table 1, entry 9). Purified by $\mathrm{SiO}_{2}$ chromatography (ether/hexanes $=1 / 24) ; R_{\mathrm{f}}=0.45$ (ether/hexanes $=1 / 9$ ); IR (neat): 2958, 1716, 1599, 1172, $819 \mathrm{~cm}^{-1} ;{ }^{1} \mathrm{H}$ NMR (400 MHz, $\left.\mathrm{CDCl}_{3}\right) \delta 0.21(\mathrm{~s}, 6 \mathrm{H}), 1.27(\mathrm{t}, J=7.2 \mathrm{~Hz}, 3 \mathrm{H}), 4.10(\mathrm{~s}, 2 \mathrm{H}), 4.17$ $(\mathrm{q}, J=7.2 \mathrm{~Hz}, 2 \mathrm{H}), 6.25(\mathrm{~s}, 1 \mathrm{H}), 7.06(\mathrm{~d}, J=7.2 \mathrm{~Hz}, 2 \mathrm{H}), 7.14-7.20(\mathrm{~m}, 3 \mathrm{H}), 7.33-7.41(\mathrm{~m}, 5 \mathrm{H}) ;{ }^{13} \mathrm{C}$ 
NMR (100 MHz, $\left.\mathrm{CDCl}_{3}\right) \delta-2.75,14.46,36.97,60.27,126.20,128.06,128.35,129.23,129.57,129.78$, 134.29, 136.62, 138.95, 160.98, 165.87; EIMS $m / z(\%)$ : 246(100), 218(39), 135(69), 115(35), 75(49); HREIMS calcd for $\mathrm{C}_{19} \mathrm{H}_{21} \mathrm{O}_{2} \mathrm{Si}$ : 309.1311; found $309.1303\left[\mathrm{M}^{+}-\mathrm{CH}_{3}\right]$.

Determination of ee. Representative procedure for derivatization. (5S)-1-[(3'S)-3-(Dimethylphenylsilyl)butanoyl]-5-(triphenylmethoxymethyl)pyrrolidin-2-one. To a solution of ethyl (3S)-3(dimethylphenylsilyl)butanoate (Table 1, entry 1; $220 \mathrm{mg}, 0.88 \mathrm{mmol}$ ) in $\mathrm{EtOH}(4 \mathrm{~mL})$ and $\mathrm{H}_{2} \mathrm{O}(1 \mathrm{~mL})$ was added $\mathrm{NaOH}(70 \mathrm{mg}, 1.75 \mathrm{mmol})$ at $\mathrm{rt}$. The mixture was stirred at $\mathrm{rt}$ for $14 \mathrm{~h}$ and then concentrated by rotary evaporation. The residue was quenched by pouring into $1 \mathrm{~N} \mathrm{HCl}$ and diluted with ethyl acetate. The aqueous layer was extracted with ethyl acetate and the combined organics were washed with brine, dried over anhydrous $\mathrm{MgSO}_{4}$, filtered, and concentrated by rotary evaporation. The residue was purified by flash chromatography (ethyl acetate/hexanes $=1 / 2$ ) to afford $(3 S)$-3-(dimethylphenylsilyl)butanoic acid (184 mg, 94\%) as a clear oil. To a solution of (3S)-3-(dimethylphenylsilyl)butanoic acid (179 $\mathrm{mg}, 0.805 \mathrm{mmol})$ in $\mathrm{CH}_{2} \mathrm{Cl}_{2}(4 \mathrm{~mL})$ was added oxalyl chloride $(140 \mu \mathrm{L}, 1.61 \mathrm{mmol})$ at $0{ }^{\circ} \mathrm{C}$. The mixture was stirred at $\mathrm{rt}$ for $2 \mathrm{~h}$ and then concentrated by rotary evaporation to afford acyl chloride. To a solution of $(S)$-5-(triphenylmethoxymethyl)pyrrolidin-2-one $(288 \mathrm{mg}, 0.81 \mathrm{mmol})$ in THF $(4 \mathrm{~mL})$ was added $n$-BuLi $(0.60 \mathrm{~mL}, 0.97 \mathrm{mmol})$ at $-78{ }^{\circ} \mathrm{C}$. The mixture was stirred at $-78{ }^{\circ} \mathrm{C}$ for $15 \mathrm{~min}$. To the reaction mixture was added acyl chloride in THF $(2 \mathrm{~mL})$ at $-78{ }^{\circ} \mathrm{C}$ and the mixture stirred at $-78{ }^{\circ} \mathrm{C}$ for $2 \mathrm{~h}$. The reaction was quenched by pouring into sat. aq. $\mathrm{NH}_{4} \mathrm{Cl}$ and diluted with ethyl acetate. The aqueous layer was extracted with ethyl acetate and the combined organics were washed with brine, dried over anhydrous $\mathrm{MgSO}_{4}$, filtered, and concentrated by rotary evaporation. The residue was purified by flash chromatography (ethyl acetate/hexanes $=1 / 4$ ) to afford the title product (194 mg, 43\%) as a clear oil; $R_{\mathrm{f}}=0.37$ (ethyl acetate/hexanes = 1/4); IR (neat): 3068, 2955, 1738, 1694, $1597,1366,1221,1112,1086 \mathrm{~cm}^{-1} ;{ }^{1} \mathrm{H}$ NMR $\left(400 \mathrm{MHz}, \mathrm{CDCl}_{3}\right) \delta 0.32$ (s, 3H), 0.33 (s, 3H), 0.89 (d, $J$ $=7.2 \mathrm{~Hz}, 3 \mathrm{H}), 1.51(\mathrm{~m}, 1 \mathrm{H}), 1.88-2.10(\mathrm{~m}, 2 \mathrm{H}), 2.45(\mathrm{ddd}, J=17.8,9.8,1.6 \mathrm{~Hz}, 1 \mathrm{H}), 2.74(\mathrm{dd}, J=16.3$, $11.4 \mathrm{~Hz}, 1 \mathrm{H}$ ), 2.89 (dd, $J=17.8,10.6 \mathrm{~Hz}, 1 \mathrm{H}), 2.94$ (dd, $J=16.3,3.1 \mathrm{~Hz}, 1 \mathrm{H}), 3.15$ (dd, $J=9.7,2.6 \mathrm{~Hz}$, $1 \mathrm{H}), 3.46(\mathrm{dd}, J=9.7,4.0 \mathrm{~Hz}, 1 \mathrm{H}), 4.43(\mathrm{~m}, 1 \mathrm{H}), 7.16-7.27(\mathrm{~m}, 9 \mathrm{H}), 7.29-7.37(\mathrm{~m}, 9 \mathrm{H}), 7.50-7.56(\mathrm{~m}$, $2 \mathrm{H}) ;{ }^{13} \mathrm{C}$ NMR $\left(100 \mathrm{MHz}, \mathrm{CDCl}_{3}\right) \delta-5.01,-4.81,14.57,15.37,21.30,33.31,39.11,56.71,64.17,87.04$, $127.23,127.87,127.97,128.63,129.14,134.14,137.78,143.70,173.94,176.24$; EIMS $m / z(\%)$ : 561(5.5)[M $\left[\mathrm{M}^{+}\right], 546(14), 318(18), 248,(32), 244(21), 243(100)$; HREIMS calcd for $\mathrm{C}_{36} \mathrm{H}_{39} \mathrm{NO}_{3} \mathrm{Si}$ : 561.2699; found 561.2701[ $\left.\mathrm{M}^{+}\right]$.

(1) Fujiwara, T.; Sawabe, K.; Takeda, T.; Tetrahedron 1997, 53. 8349. 